\title{
Folding Dynamics of Single GCN-4 Peptides by Fluorescence Resonant Energy Transfer Confocal Microscopy
}

Yiwei Jia ${ }^{1}$, David S. Talaga ${ }^{1}$, Wai Leung Lau ${ }^{2}$, Helen S. M. Lu², William F. DeGrado², Robin M. Hochstrasser ${ }^{1}$

${ }^{1}$ Department of Chemistry and ${ }^{2}$ Department of Biophysics and Biochemistry University of Pennsylvania, Philadelphia, PA 19104

\begin{abstract}
We have prepared a bichromophoric crosslinked variant of GCN4-P1 for single molecule fluorescence energy transfer experiments (GCN4-Pf). The folding and unfolding fluctuations of single GCN4-Pf molecules are measured in a two channel confocal microscope with which donor and acceptor fluorescence trajectories are measured simultaneously. The energy transfer efficiency is thereby determined and its probability distributions as a function of added denaturant [urea] are calculated. The distributions indicate that single molecule GCN4-Pf is in dynamic folding equilibrium with the position of the equilibrium being altered by the concentration of urea.
\end{abstract}




\section{Introduction}

Single molecule measurements are now moving from the realm of technological demonstrations to making contributions to new understanding of chemical systems particularly those that are microscopically inhomogeneous and exhibit dynamics over many time scales.[1-9] Using single molecule detection, it is now possible to follow the evolution in time of individually selected members of an equilibrium ensemble. This trajectory can be used to evaluate rates, rate constants, and distributions of other properties. On the other hand, bulk measurements usually give only ensemble averaged value of the molecular property in question.

Proteins and other biological assemblies exhibit microscopic structural heterogeneity and are therefore of particular interest for single molecule studies. The structural fluctuations of proteins can result in folding and unfolding of the primary sequence of amino acids between a well defined three-dimensional native structure and a broadly distributed set of denatured structures. [4,10-14] In one example, fluorescence spectral fluctuations were attributed to dynamics amongst protein substrates in single molecule experiments.[6] In another study, protein conformational dynamics was cited as the origin of fluorescence intensity and polarization fluctuations of single Staphylococcal nuclease.[4]

This paper concerns the study of a peptide derived from the yeast transcription factor, GCN4. The DNA binding domain of this protein includes a sequence that forms a short segment of a two-stranded coiled coil $[15,16]$, as shown in figure 1 . Since their discovery, coiled coils have provided very simple model systems for the study of the folding of water-soluble proteins.[12,13,17] A peptide spanning the coiled coil of GCN4 
(GCN4-P1) has been shown to form a cooperatively folded helical dimer. [12,13,18-22].

This peptide is an excellent system for studying protein folding because it is quite simple, and yet contains a well-packed helix/helix interface, as found in globular proteins. It has been shown to exist in a two-state equilibrium between unstructured monomers and fully alpha-helical dimers ${ }^{12}$. The alpha-helical secondary structure and the double-helical folded structure apparently form concomitantly.[13,17-20,23] To simplify the folding reaction, Kim and coworkers have introduced a covalent disulfide tether between the two-peptide chains. The crosslink stabilizes the proteins towards thermal denaturation, although the peptide continues to fold in an apparent, two-state equilibrium. [24]

One purpose of this study is to investigate the microscopic features of a macroscopically observed kinetic model. GCN4-P1 exhibits two-state folding kinetics when in bulk solution $[17,20]$, although a folding intermediate has occasionally also been observed for some coiled coils [13,19,23]. It has been shown, however, that macroscopic averaging can create effective two-state kinetics in the bulk even when there are multiple unfolded states of the protein.[10]

Single molecule experiments are sensitive to mechanistic heterogeneity. Since single molecule experiments are immune to ensemble averaging, it is possible, in principle, to distinguish between a pathway-based folding mechanism and a landscape multi-path mechanism. If a protein has a single folding pathway, then the distribution of observed kinetic rates should be narrow. Conversely if there are multiple pathways there would, in general, be multiple rates associated with folding and an appropriate distribution of rates would be observed in a single molecule experiment. 


\section{Modifications to GCN4}

The GCN4-P1 variant employed in this study, designated GCN4-Pf, has the sequence

GGRMKQLED ${ }^{10}$ KVEELLSKDY $^{20}{ }^{\text {HLENEVARL }}{ }^{30}$ KKLVGERGG $^{40}$ CGEEEEE. Its design incorporates several distinct features (Fig. 1): Asn-18, which forms a buried hydrogen bond with Asn-18' of a neighboring helix in GCN4-P1, has been changed to Asp in GCN4-Pf. The presence of aspartic acid at the helix/helix interface of the peptide introduces a "pH switch," which destabilized the peptide at high $\mathrm{pH}$, allowing the modulation of stability with $\mathrm{pH}$ as well as urea.[25] Five glutamic acid residues were also appended to the $\mathrm{C}$-terminus, providing a flexible appendage to allow electrostatic adsorption of the peptides onto a positively charged surface for single molecule studies. A Gly-Gly-Cys-Gly tetrapeptide was inserted between GCN4-P1 and the polyglutamic acid tail. Crosslinking of the two helices by a disulfide bond formation at the Cys residue provides an unimolecular-folding situation. This simplification is of particular importance given the low concentrations $\left(<10^{-11} \mathrm{M}\right)$ used for single molecule experiments where the likelihood of observing an "on" bimolecular folding event would otherwise be vanishingly small. The Gly residues serve as a flexible linker between GCN4-P1 and the polyglutamic acid tail.

In order to probe conformational fluctuations in the folded and unfolded states we synthesized GCN4-Pf with a fluorescence donor and acceptor pair attached to the Ntermini. Initially, two different reduced cysteinyl peptides were prepared with the same peptide sequence but different N-terminal fluorophores that form an energy transfer pair. Texas Red-X (TxR) behaves as the energy acceptor while 5-carboxyrhodamine 6G (R6G) 
behaves as the energy donor. These fluorescently labeled monomers were first reduced, then recombined under oxidative conditions to form the desired heterodimeric peptide.

\section{Experimental}

\section{Materials}

Abbreviations used: APS, aminopropylsilanyl; EDTA, ethylenediaminetetraacetic acid; HOBT, 1-hydroxybenzotriazole; Fmoc, 9-fluorenylmethoxycarbonyl; MES, 2-(Nmorpholino)ethanesulfonic acid; $\mathrm{KCl}$, potassium chloride; $\mathrm{R} 6 \mathrm{G}$, rhodamine-6G; TxR, Texas red; TFA, trifluoroacetic acid; Tris, tris(hydroxymethyl)aminomethane; MRE, mean residue ellipticity.

(3-Aminopropyl)trimethoxysilane was purchased from Lancaster synthesis, Inc.. Reagents for peptide synthesis were purchased from Advanced Chem Tech, Inc. HOBT, piperidine, and picric acid were obtained from Aldrich. Solvents were obtained from J. T. Baker. Tris, EDTA, $\mathrm{KCl}$ and TFA were purchased from Sigma. Amine reactive dyes were purchased from Molecular Probes: R6G \#C-6127; TxR \#T-6134)

\section{Synthesis and Characterization of GCN-Pf}

The GCN4-Pf polypeptide was synthesized using a standard solid-phase Fmoc method on a PE Applied Biosystems 433A peptide synthesizer. The fluorophores were coupled to the N-terminal amino group following assembly of the peptide on the solid support. The N-terminal Fmoc group was deprotected by $20 \%$ piperidine to generate a free amino terminus, and then coupled with approximately 1 equivalent of Texas Red-X N-hydroxysuccinimide ester (or 5-carboxyrhodamine 6G N-hydroxysuccinimide ester) and HOBT, 10 equivalents of diisopropylethylamine in dimethylformamide. The reaction was conducted under argon in the dark for 48 hours. Following cleavage from the resin 
with trifluoroacetic acid with thioanisole, ethanedithiol and anisole in the 90:5:3:2 (v/v), the peptides were purified to homogeneity by reverse-phase HPLC using a Vydac C18 column (22x250 mm 15-20 micron particle size) running a linear acetonitrile-water gradient (with segments of $0.25 \%$ by volume increase per minute of acetonitrile) in the presence of $0.1 \%$ TFA. If necessary the Cys residue was first reduced to the thiol by reaction with tris[2-carboxyethyl]phosphine.

The GCN4-Pf heterodimer was prepared by cross-linking the individual 5carboxyrhodamine 6G and Texas Red labeled peptides via a disulfide bond. Equal amount of the two peptides $(1.1 \mathrm{mg} / \mathrm{ml})$ were reacted in $0.25 \mathrm{mM}$ (reduced/oxidized 1:1 ratios) glutathione, $0.1 \mathrm{M} \mathrm{KCl,} 0.1 \mathrm{M}$ Tris $\mathrm{pH} 8.5$ and $1 \mathrm{mM}$ EDTA. Cysteine oxidation resulted in the formation of two different homodimers and the desired heterodimer. Peptide mixtures were fractionated by reverse-phase HPLC using a Vydac C18 column (22x250 mm 15-20 micron particle size). The peptides were eluted by means of a linear gradient from 33 to $45 \%$ of acetonitrile in TFA $0.1 \%$ over 52 minutes; elution was monitored at $222 \mathrm{~nm}$. The identity of the heterodimer was confirmed by MALDI-TOF mass spectrometry using a Perspective Biosystems Voyager DE-RP BioSpectrometry Workstation. A singly labeled GCN-4 with donor only was prepared by a modification of this procedure.

\section{CD spectroscopy of GCN4-Pf heterodimer in solution}

Circular dichroism (CD) spectra of fluorescently labeled GCN4-Pf was measured in $10 \mathrm{mM}$ MES, pH 6.1 at a total peptide concentration of approximately $2 \mu \mathrm{M}$ in a $1 \mathrm{~mm}$ pathlength cell. The peptides showed the double minima at 208 and $222 \mathrm{~nm}$ characteristics of alpha helices. 
Aminopropylsilanization (APS) of quartz and glass surface

Clean glass or quartz slides $(2 \times 1 \times 0.4 \mathrm{~cm})$ were amino-silanized by reaction with $0.1 \%(\mathrm{v} / \mathrm{v})(3$-aminopropyl)trimethoxysilane in hexane, providing a surface that was positively charged at neutral $\mathrm{pH}$ or below. The reaction was allowed to proceed for one hour at room temperature. To determine surface density of free amine on the modified quartz, the plates were immersed in $0.1 \mathrm{M}$ picric acid in dichloromethane, and then washed with the same solvent. This procedure resulted in formation of chromophoric picrate salts of the aminopropyl groups on the derivatized surface[26]. The slides were then placed inside a cuvette filled with dichloromethane. The quartz slides were maintained in vertical position by two custom-made Teflon spacers. The absorbance at $358 \mathrm{~nm}$ was used to estimate the surface density of amines, using an extinction coefficient of picrate of $14,500 \mathrm{M}^{-1} \mathrm{~cm}^{-1}$. The free amino form of the derivatized surface was regenerated by washing the slides with 5\% DIEA (v/v) in DCM and then DCM. The amount of picrate eluted into solution in this manner agrees well with the amount detected directly on the surface. The quartz slides were used within 24 hours. The degree of modification thus determined was approximately one amine per $20 \AA^{2}$, indicative of a single monolayer of well-packed monomers.

For single molecule studies, microscope cover slips (Fisher, No. 1.5) are modified as above. GCN4-Pf is adsorbed to the surface by applying $30 \mu \mathrm{l}$ of a $1.0 \mathrm{nM}$ solution of GCN4-Pf in $10 \mathrm{mM}$ MES pH 6.1 buffer for 5 minutes. The surface is then washed three times, then loaded with the same buffer containing the desired urea concentrations. 
Circular dichroism of monolayers of GCN4-Pf on APS-modified quartz

GCN4-Pf heterodimer was dissolved in $10 \mathrm{mM}$ MES pH 6.1 and then transferred to a $1 \mathrm{~cm}$ pathlength quartz cuvette containing four APS-modified quartz slides held in a vertical position by two custom-made Teflon spacers. The peptide was added at a concentration of $4.6 \mu \mathrm{M}$, providing an approximately 5-fold excess of the peptide relative to the amount required to form a uniform monolayer of the peptides in a vertical orientation. The incubation time was one hour. The quartz slides inside the cuvette were rinsed once prior to filling the cuvette with known volume of $10 \mathrm{mM}$ MES pH 6.1 buffer. Circular dichroism spectra were acquired on an Aviv 62A DS or 62A circular dichroism spectrophotometer. Far-UV spectra were scanned from 300 to $195 \mathrm{~nm}$. The amount of surface-adsorbed peptide was calculated from its absorbance at $520 \mathrm{~nm}$, using an extinction coefficient of 108,000 mmole/ $\mathrm{cm}^{2}$ (as given by Molecular Probes, Inc). The observed density of peptide on the surface was $8 \mathrm{~nm}^{2} /$ dimeric molecule. By comparison, the cross-sectional area of the GCN4 coiled coil in crystals is $6.6 \mathrm{~nm}^{2} /$ dimeric molecule.[15]

As compared with the sample in homogeneous solution, the immobilized GCN4 peptide shows a similar CD spectrum, except the minimum at $208 \mathrm{~nm}$ is of lower intensity (Figure 2). This finding suggests that the helices may be oriented perpendicular to the quartz and parallel to the incident UV light, based on the known polarization of this $\pi-\pi^{*}$ transition.[27]

A urea denaturation curve for surface-adsorbed GCN4-Pf was determined by monitoring the change in $[\theta]_{222}$ as a function of urea concentration. At each urea concentration, data were collected and averaged for two minutes. To facilitate mixing of 
the aqueous contents, only two peptide-covered quartz slides were used in the urea denaturation experiment. The data in Fig. 3 represent the average of three experiments. The urea-induced denaturation of the peptide exhibits an unfolding transition midpoint around $3 \mathrm{M}$ urea. This value is similar to that observed in bulk solution (data not shown), indicating that the quartz surface did not greatly perturb the peptide's conformational properties.

\section{Single Molecule Apparatus}

The home-built inverted scanning confocal microscope has been described before.[9] To excite the single molecules we used a 76MHz mode-locked Nd:YAG laser (Coherent Antares) frequency doubled to $532 \mathrm{~nm}$. An average excitation power of 0.5 $\mu \mathrm{W}$ is coupled into the microscope by a dichroic mirror (550 DCLP, Chroma). The excitation light is made circularly polarized by a $\lambda / 4$ waveplate. The reflected and scattered $532 \mathrm{~nm}$ light is removed by a $532 \mathrm{~nm}$ holographic filter (Notch Plus, Kaiser). The fluorescence photons of R6G and TxR are separated by a second dichroic mirror (580 DCLP, Chroma). Further rejection of background and crosstalk is achieved by filtering the fluorescence of R6G with a band pass filter (540-580 nm, Chroma) and a long pass filter (OG550, Schott). Similarly, a long pass filter (OG590, Schott) and a band pass filter (600-660 nm, Chroma) are also used for the detection TxR Fluorescence. Two Avalanche photodiodes (EG\&G) provide simultaneous high quantum yield detection of single photons from each of the fluorescent dyes. Fluorescence images of GCN-4 labeled with both R6G and TxR were recorded simultaneously by scanning the sample stage (area: $36 \mu \mathrm{m}^{2}$ ). Photo-bleaching curves of R6G and TxR were simultaneously recorded with an integration time of $0.98 \mathrm{~ms}$. 


\section{Results}

Macroscopic Characterization of GCN4-Pf in Solution on APS-quartz

The goal of the present paper is to measure conformational fluctuations of GCN4Pf in the folded and unfolded states, as well as under conditions where it is in dynamic equilibrium between these two conformational ensembles. We therefore first measured the folding equilibrium of GCN4-Pf, both in solution and adsorbed to APS-quartz. The CD spectrum of GCN4-Pf (Figure 1) in aqueous solution and on APS-quartz is typical of that of $\alpha$-helical proteins. Preliminary solution experiments showed that the folded conformation of GCN4-Pf was increasingly destabilized as the $\mathrm{pH}$ was increased from 3 to 8 . The current studies were conducted at $\mathrm{pH} 6.1$, because this value provided a marginal degree of stability favoring the folded form.

Figure 3 illustrates a urea denaturation curve for surface-adsorbed GCN4-Pf, as monitored by CD spectroscopy. The curve is similar to that observed for the peptide in free solution, and shows a midpoint near 3.0 M urea. The urea-induced denaturation curves of small proteins are generally analyzed assuming a two-state equilibrium involving a folded and unfolded ensemble of conformers. The free energy for this process, $\Delta \mathrm{G}_{\text {fold }}$, has been shown to scale approximately linearly with respect to the concentration of urea. Application of this model to the data in Fig. 3 indicated that the value of $\Delta \mathrm{G}_{\text {fold }}$ (extrapolated to $0 \mathrm{M}$ urea) was approximately $-1.6 \pm 0.4 \mathrm{Kcal} / \mathrm{mol}$ (midpoint $=2.8 \pm 0.3 \mathrm{~m}$ urea), although those values are somewhat uncertain because the folded and unfolded baselines were not well resolved. Thus, GCN4-Pf is approximately $90 \%$ folded in the absence of urea at $\mathrm{pH} 6.1$. 


\section{Images}

Single molecule fluorescence images of GCN4-Pf at $\mathrm{pH} 6.1$ in the absence of urea are shown in Fig. 4. With $532 \mathrm{~nm}$ excitation, mainly the R6G is excited but the TxR channel shows significantly more fluorescence spots. In addition, the intensity of the emission of TxR is stronger than R6G. These results are consistent with the protein system being mainly in a folded state at $\mathrm{pH} 6.1$ in the absence of urea; the donor-toacceptor distance is relatively close (Fig. 1), and the energy transfer efficiency is high under these conditions. Fig. 5 shows single molecule fluorescence images at pH 6.1 and 7.4M urea. In this case, the R6G channel shows significantly more emission, indicating less effective energy transfer and an unfolded condition.

\section{Dual Channel Detection}

The overlap between the donor and acceptor emission spectra influences the signals seen in each channel. To correct for this cross-talk we note that the overall signal measured at each detector $\left(\mathrm{S}_{\mathrm{D}}\right.$ and $\left.\mathrm{S}_{\mathrm{A}}\right)$ is a linear combination of true donor and acceptor intensities $\left(\mathrm{I}_{\mathrm{D}}\right.$ and $\left.\mathrm{I}_{\mathrm{A}}\right)$ and the observed background for each channel $\left(\mathrm{B}_{\mathrm{D}}\right.$ and $\left.\mathrm{B}_{\mathrm{A}}\right)$.

$$
\begin{aligned}
& S_{D}=C_{D D} I_{D}+C_{D A} I_{A}+B_{D}, \quad S_{A}=C_{A A} I_{A}+C_{A D} I_{D}+B_{A} \\
& I_{A}=\frac{C_{D D}\left(S_{A}-B_{A}\right)-C_{A D}\left(S_{D}-B_{D}\right)}{C_{D D} C_{A A}-C_{A D} C_{D A}}, \quad I_{D}=\frac{C_{A A}\left(S_{D}-B_{D}\right)-C_{D A}\left(S_{A}-B_{A}\right)}{C_{A A} C_{D D}-C_{D A} C_{A D}}
\end{aligned}
$$

The coefficients $C_{i j}$ represent the amount of $i(i=A, D)$ signal reaching channel $j$ $(\mathrm{j}=\mathrm{A}, \mathrm{D})$ and are determined experimentally using two different methods. We used the fluorescence of singly labeled GCN4-Pf molecules to measure the cross-talk between channels. During the course of the measurements of the GCN4-Pf we also measured the cross-talk after the acceptor molecule became bleached. These measurements allow the individual trajectories to be corrected to obtain signals proportional to the donor and the 
acceptor fluorescence intensities. There is no appreciable leakage of the acceptor signal into the donor detection channel.

\section{Types of trajectories}

Fig. 1 illustrates typical single-molecule recordings of the variation in fluorescence intensity with respect to time for GCN4-Pf. We observe a number of different types of trajectories. The trajectories in figure 6 are representative of those acquired for GCN4-Pf. The trajectory in figure 6(a) is typical [i.e., occurs ca. $70 \%$ of the time] of those providing strong evidence for energy transfer between the donor and acceptor. The acceptor signal dominates until it photobleaches to generate a state that can no longer act as an excitation energy acceptor. At that time, the donor signal jumps to its non-perturbed level indicating no energy transfer. We interpret the abrupt termination of the signal as photobleaching; it should not be confused with a folding/unfolding event. The abruptness and completeness of this transition indicates that it is a single molecule event. Another class of trajectories [ca. 25\%] is shown in Figure 6(b), in which, within the time resolution of the experiment, the acceptor and donor bleach simultaneously. Sometimes the donor bleaches first [ca. 5\%] and we see a reduction of the acceptor signal to the level of its direct excitation, as in Figure 6(c). Figure 7 shows a single molecule trajectory on an expanded time scale which more clearly shows the anticorrelated fluctuations in the donor and acceptor signals. The anticorrelated signal was absent from GCN4-Pf molecules labeled with only the donor, indicating the detection channels are uncoupled. Furthermore, the mean squared signal fluctuations (excluding shot noise) were ca. 6 times less for the single labeled peptide. Thus, most of the variance in the 
trajectories arises from variations in the donor/acceptor distances, the angles between donor and acceptor transition dipoles and the orientation of the dyes relative to the surface.

\section{Sources of fluorescence intensity fluctuations}

Single molecule fluorescence intensity fluctuations in our experiments can arise from a variety of sources. We are focused on extracting the fluctuations related to stochastic motions in the folded and unfolded states, as well as the dynamics of interconversion between those two states. Photophysical processes that may also give rise to intensity fluctuations include time-dependent shifts in the fluorescence spectrum[28,29], transitions in and out of non-fluorescent states of the system[30], including triplet states[31,32] and irreversible photobleaching[1]. We eliminated the contributions of some photophysical processes. The GCN4 labeled with only R6G or TxR did not exhibit any cross correlations of the signal between the two channels, indicating that fluctuations of the fluorescence spectral positions are not contributing to this signal. Triplet lifetimes in the presence of $\mathrm{O}_{2}$ should be on the order of microseconds. We deliberately avoided the use of an $\mathrm{O}_{2}$ scavenging system[2] to minimize complications from transitions to triplet states that might otherwise complicate our interpretation of the millisecond signal fluctuations. Finally, following photobleaching, the trajectories exhibit long-lived non-fluorescent states; these latter portions of the trajectories were not included in our analysis.

Angular motions of the transition dipoles of the probes R6G and TxR can also contribute to the fluctuations. Because we use circularly polarized excitation and the detection system is not polarization sensitive, the variations in the azimuthal angles, $\Phi$, 
do not influence the signal intensity. However, variations in the colatitude, $\theta$, of both donor and acceptor can affect the measured intensities. In independent experiments on R6G or TxR bound to a GCN4-P1 peptide we measured the decay of the fluorescence anisotropy. In both cases, the anisotropy of the probes relaxed on a subnanosecond time scale exhibiting an order parameter of ca. 0.5 . The total anisotropy decayed in 2-3 ns. These results indicate fast rotational motion of the probes relative to the peptide and significant rotational averaging of the energy transfer signals is occurring on the nanosecond time scale. The relative signal detected from a radiating dipole having colatitude $\theta$, is within a few percent of $\sin ^{2} \theta\left(1+\sin ^{2} \theta\right)$ for our microscope, when the excitation is circularly polarized[33]. Therefore the relative signal intensities from donor and acceptor depend not only on the angles involved in the dipole-dipole interaction that form part of the energy transfer efficiency, but also on the colatitudes $\theta_{\mathrm{A}}$ and $\theta_{\mathrm{D}}$. Therefore the signal will also contain contributions from fluctuations in these angles.

\section{Calculation of the energy transfer quantum yield}

The corrected count rates are used with the donor and acceptor extinction coefficients to determine the quantum yield for energy transfer.

$$
\begin{aligned}
& I_{D}=\varepsilon_{D} \frac{\emptyset_{D}\left(1-\emptyset_{E T}\right)}{1-\emptyset_{E T}\left(1-\emptyset_{D}\right)} \\
& I_{A}=\emptyset_{A}\left(\varepsilon_{A}+\varepsilon_{D} \frac{\emptyset_{D} \emptyset_{E T}}{1-\emptyset_{E T}\left(1-\emptyset_{D}\right)}\right) \\
& \emptyset_{E T}=\frac{I_{A} \varepsilon_{D} \emptyset_{D}-I_{D} \varepsilon_{A} \emptyset_{A}}{\varepsilon_{D} \emptyset_{D}\left(I_{A}+I_{D} \emptyset_{A}\right)}=\frac{I_{A} \emptyset_{D}-I_{D} \varepsilon_{A / D} \emptyset_{A}}{\emptyset_{D}\left(I_{A}+I_{D} \emptyset_{A}\right)}
\end{aligned}
$$


Where $\emptyset_{D}, \emptyset_{A}, \varnothing_{E T}$ are the quantum yields for unsensitized donor and acceptor fluoresence and energy transfer, respectively. $\varepsilon_{D}, \varepsilon_{A}, \varepsilon_{A \mid D}$ are the extinction coefficients of the donor and the acceptor and their ratio at the excitation wavelength. $I_{D}, I_{A}$ are the corrected measured intesities of the donor and the acceptor fluorescence from the single molecule trajectories. Assuming the Förster mechanism for the energy transfer[34], the yield $\emptyset_{\mathrm{ET}}$ for a distance $\mathrm{R}$ between chromophores is:

$$
\emptyset_{\mathrm{ET}}=\left(1+\left(\frac{\mathrm{R}}{\mathrm{R}_{0}}\right)^{6}\right)^{-1}
$$

where $\mathrm{R}_{0}$ is the distance between chromophores that gives a quantum yield for energy transfer of 50\%. The factor $\mathrm{R}_{0}$ depends on the spectral overlap of donor emission and acceptor absorption and on the angular part of the transition dipole-dipole interaction.

\section{Distributions of energy transfer efficiency}

For a given time point in a trajectory we average $2 n+1$ points ( $n$ before and $n$ after, in addition to the point) to obtain an observed count rate for the donor and the acceptor. The number of points averaged determines the time resolution of the distribution. We use $\mathrm{n}=1$ for $3 \mathrm{~ms}, \mathrm{n}=12$ for $25 \mathrm{~ms}$, and $\mathrm{n}=50$ for $100 \mathrm{~ms}$ time resolution when measuring the distributions of energy transfer efficiency and deducing the donoracceptor distance distribution.

In Figure 8 the results of 60-70 trajectories are averaged to compute an overall distribution of energy transfer efficiency for each concentration of denaturant. The distribution is narrow and peaked at $\sim 97 \%$ for $0 \mathrm{M}$ urea. The distribution broadens considerably and the peak moves to $\sim 90 \%$ for the midpoint of the urea denaturation curve at $3 \mathrm{M}$ Urea. At $7.4 \mathrm{M}$ urea the peak of the distribution is at $\sim 80 \%$ and further broadens. 


\section{Position distributions}

Figure 9 shows the distributions $\mathrm{P}_{\mathrm{F}}(\mathrm{R})$ and $\mathrm{P}_{\mathrm{U}}(\mathrm{R})$ of the average donor/acceptor distances for the folded (F) and unfolded (U) states calculated assuming an $\mathrm{R}^{-6}$ distance dependence of the energy transfer rate and an angular factor $k^{2}=2 / 3$. The function $P_{F}(R)$ is obtained by subtracting $10 \%$ of $\mathrm{P}_{\mathrm{U}}(\mathrm{R})$ from the $0 \mathrm{M}$ urea distribution. As the urea concentration increases, the average distance between chromophores and the variance of the distribution calculated with these assumptions increases. Similarly the overall width of the distance distribution for individual molecules increases with denaturant

concentration (data not shown). The peak of the distribution for $\mathrm{P}_{\mathrm{U}}(\mathrm{R})$ occurs at $25-30 \AA$, which is somewhat longer than the value expected from the design. Models of GCN4-Pf based on the crystal structure of GCN4-P1[15] would predict a distance of approximately 15-20 A. This discrepancy is possibly due to unwinding the ends of the helices. Furthermore there are systematic errors in the calculation of the energy transfer efficiency due to the distribution of $\theta$ angles.

\section{Discussion}

Relationship of single molecule measurements to bulk measurements

The energy transfer quantum yield distributions in Figure 8 suggest that the protein is most ordered at $0 \mathrm{M}$ urea, and less ordered at $7.4 \mathrm{M}$ urea, where the distribution is broader, with $3 \mathrm{M}$ urea being intermediate between the two. This conclusion is consistent with our understanding of the bulk folding experiments and directly leads to properties of the folded and unfolded states of the protein. At $\mathrm{pH} 6.1$ the protein is not under completely native conditions. Therefore, even at $0 \mathrm{M}$ urea some fully unfolded structures will be populated, assuming that the peptide folds in an effectively two-state process. 
We have considered the effect of the angular distribution on the energy transfer distribution. From the fluorescence anisotropy measurements we showed that the rotational motions of the donor and acceptor are nanosecond processes. In the single molecule experiment, each photon we detect arises from a particular separation, $\mathrm{R}$, and orientation, $\Omega$, of the donor and acceptor. The energy transfer efficiency was measured by averaging ca. 6 photons per ms. So that at the shortest time resolution of $3 \mathrm{~ms}$ we collect on average 18 photons. If we assume that $\mathrm{R}$ and $\Omega$ are statistically independent and accept that the rotational motions occur over the whole range of $\Omega$ and are much faster than the measurement, even 18 measurements approximately averages the angular factor of the Förster theory to $2 / 3$. This averaging should be much more complete for the $100 \mathrm{~ms}$ time resolution distributions. We will also assume that the signal is effectively averaged over the colatitudes $\theta_{\mathrm{A}}$ and $\theta_{\mathrm{D}}$ that were discussed earlier as a possible source of signal fluctuations. In future experiments, we expect to sharpen our knowledge of these effects by analyzing the signals in polarized light. With the assumption of rotational averaging we can convert the energy transfer efficiency distributions into the distance distributions in Figure 9. The overall similarity of the distributions at different time resolutions shows that the distance distribution is not completely averaged on the measurement timescale.

The low $\emptyset_{E T}$ tail present in all the probability distributions increases in amplitude as the denaturant concentration is increased. This tail is also present in the distributions calculated from unaveraged trajectories from single molecules. Since individual molecules can exhibit the entire range of configurations, the distributions (see Figure 8) averaged over many trajectories must have a dynamical component. 


\section{Possible models that explain observed distributions}

The bulk equilibrium may be described in terms of two states, one with a large and the other with a small degree of helicity. However the single molecule experiments are not expected to exhibit fluctuations between only two values of the energy transfer yield. A range of energy transfer efficiencies would be a natural consequence of different configurations being created each time the protein unfolds on the silanized glass surface. In addition, there will be fluctuations of the structure within each thermodynamic state that will cause energy transfer changes.

Our results clearly show that the distribution of energy transfer efficiencies and of donor acceptor distances is quite different in $0 \mathrm{M}$ and $7.4 \mathrm{M}$ urea. We will refer to these distributions as the folded and unfolded distributions. They may be close to the equilibrium distributions for the two states although we would expect that there are also rapid dynamic processes that are averaged on the ms time scale. Furthermore we obtain the distribution function of $\mathrm{R}$ and we do not know the relationship between $\mathrm{R}$ and the other coordinates of the protein. Nevertheless a picture of folding in this limited space emerges, in which we have two states with quite different "folded" and "unfolded" potentials of mean force, $\mathrm{V}_{\mathrm{F}}(\mathrm{R})$ and $\mathrm{V}_{\mathrm{U}}(\mathrm{R})$, corresponding to the two probability distributions, $\mathrm{P}_{\mathrm{F}}(\mathrm{R})$ and $\mathrm{P}_{\mathrm{U}}(\mathrm{R})$, that we measured (see Figure 10). These potentials incorporate the effects of the surface to which the protein is bound.

Our data also suggest that the rates of folding and unfolding depend on the donor acceptor distance. If we adjust the urea concentration to the midpoint of the denaturation curve the distribution observed should depend upon the relationship between the folding time and the observation time required for the measurement. When the observation time is much less than the folding-fluctuation time, the distribution is expected to be a linear 
sum of the folded and unfolded distributions. The weights of folded and unfolded components of the distribution should be equal if the molecule's environment is exactly at the midpoint of the titration curve.

Examination of the distribution observed in $7.4 \mathrm{M}$ urea with averaging times of 3 , 25, and $100 \mathrm{~ms}$ (bottom panel) are uniformly broad. Thus, there is a broad distribution of surface-associated unfolded states with different end-to-end distances that interconvert slowly on the $100 \mathrm{~ms}$ time scale. Similarly, as the averaging time varies for the $0 \mathrm{M}$ urea sample, the distribution shows a relatively small change. To examine the rates of interconversion of folded and unfolded ensembles we examined linear combinations of the 0 and $7.4 \mathrm{M}$ distributions, to determine whether they accurately predict the distribution observed at $3 \mathrm{M}$ urea. Clearly as the averaging time changes from 3 to $100 \mathrm{~ms}$, the fit to the data is increasingly poor. Thus, some interconversion of the unfolded and folded ensembles appears to occur on the 3-25 ms time scale. More specifically, the solid line in the center panel of figure 8 is the linear combination of $50 \%$ of the $0 \mathrm{M}$ distribution and $50 \%$ of the $7.4 \mathrm{M}$ distribution. For the $100 \mathrm{~ms}$ time resolution data, the $3 \mathrm{M}$ distribution does not have enough probability density at high $\emptyset_{E T}$ to fit directly to any linear combination of the $0 \mathrm{M}$ and $7.4 \mathrm{M}$ distribution functions. Reducing the averaging time to $25 \mathrm{~ms}$ improves the agreement of the linear combination of $0 \mathrm{M}$ and $7.4 \mathrm{M}$ to the $3 \mathrm{M}$ data particularly in the tail of the distribution which now seems consistent with the two-state model distribution. However the distributions still do not match well in the $90 \%$ region. This implies that the fluctuations resulting in the differences in the tail of the distribution observed at $100 \mathrm{~ms}$ averaging time are, on average, slower than $25 \mathrm{~ms}$. Reducing the averaging time to $3 \mathrm{~ms}$ results in a much better agreement between the $3 \mathrm{M}$ urea 
distribution function and the linear combination of the $0 \mathrm{M}$ and 7.4M distribution

functions. We could obtain a good least squares fit of the $3 \mathrm{M}$ data to a linear combination of the $0 \mathrm{M}$ and $7.4 \mathrm{M}$ data with weights 0.46 and 0.54 respectively. We conclude from the results that fluctuations which are changing the energy transfer efficiency from $\sim 97 \%$ to $\sim 90 \%$ when changing the urea concentration from 0 to $3 \mathrm{M}$ occur on a time scale which is between $3 \mathrm{~ms}$ and $25 \mathrm{~ms}$. The low $\emptyset_{E T}$ portion of the distribution at $25 \mathrm{~ms}$ resolution still matches well the higher resolution data, implying that fluctuations involving those values of $\emptyset_{E T}$ are still slower than $25 \mathrm{~ms}$. This result shows that there is a distribution of time scales for fluctuations in the energy transfer efficiency and a correlation between kinetics and structure. The time scales are structurally correlated such that faster time scales correspond to fluctuations that modulate $\emptyset_{E T}$ in the $90 \%$ region while the slower time fluctuations modulate $\emptyset_{E T}$ in the $70 \%$ and below region. The fluctuations that result in low $\emptyset_{E T}$ values $(<80 \%)$ are occurring on a time scale that is slower than $25 \mathrm{~ms}$.

Fluctuations in the $\emptyset_{E T} \sim 90 \%$ region are occurring faster than $25 \mathrm{~ms}$.

The separation of time scales established in this work suggests models for the GCN4-Pf/silanized glass folding kinetics. A broad distribution of folding/unfolding rates connecting two potentials $V_{F}(R)$ and $V_{U}(R)$ is indicated. Larger $R$ values correspond to slower rates. The result is that the waiting times between folding and unfolding events is not exponentially distributed. This latter point was experimentally verified and will be presented in a future publication along with the time correlations between donor and acceptor emission [35].

This work illustrates the potential of single molecule experiments to measure the distribution of molecular properties in a fluctuating and/or heterogeneous system. Not 
only could the degree of heterogeneity be determined, but also the nature and time scales

of the associated fluctuations.

\section{References}

1 X. S. Xie and J. K. Trautman, Annu. Rev. Phys. Chem. 49 (1998) 441-480.

2 T. Funatsu, Y. Harada, M. Tokunaga, K. Saito, and T. Yanagida, Nature 374 (1995) 555.

3 E. Geva and J. L. Skinner, Chem. Phys. Lett. 288 (1998) 225.

4 T. Ha, A. Y. Ting, J. Liang, W. B. Caldwell, A. A. Deniz, D. S. Chemla, P. G. Schultz, and S. Weiss, Proc. Natl. Acad. Sci. U. S. A. 96 (1999) 893-898.

5 A. Ishijima, H. Kojima, T. Funatsu, M. Tokunaga, H. Higuchi, H. Tanaka, and T. Yanagida, Cell 92 (1998) 161.

6 H. P. Lu, L. Xun, and X. S. Xie, Science 282 (1998) 1877-1882.

7 D. M. Warshaw, E. Hayes, D. Gaffney, A.-M. Lauzon, J. Wu, G. Kennedy, K. Trybus, S. Lowey, and C. Berger, Proc. Natl. Acad. Sci. U. S. A. 95 (1998) 80348039.

8 L. M. Ying and X. S. Xie, J. Phys. Chem. B 102 (1998) 10399.

9 Y. Jia, S. Sytnik, L. Li, S. Vladimirov, B. S. Cooperman, and R. M. Hochstrasser, Proc. Nat. Acad. Sci. USA (1997)

10 R. Zwanzig, Proc. Natl. Acad. Sci. U. S. A. 94 (1997) 148-150.

11 W. A. Eaton, V. Munoz, Thompson, P. A. Henry, and J. E. R. Hofrichter, Acc. Chem. Res. 31 (1998) 745.

12 K. J. Lumb, C. M. Carr, and P. S. Kim, Biochemistry 33 (1994) 7361.

13 H. Wendt, C. Berger, A. Baici, R. M. Thomas, and H. R. Bosshard, Biochemistry 34 (1995) 4097-107.

14 N. D. Socci, J. N. Onuchic, and P. G. Wolynes, Proteins: Struct. Funct. Genet. 32 (1998) 136.

15 E. K. O'Shea, J. D. Klemm, P. D. Kim, and T. Alber, Science 254 (1991) 539.

16 T. E. Ellenberger, C. J. BRANDL, K. STRUHL, and S. C. HARRISON, Cell 71 (1992) 1223-1237.

17 T. R. Sosnick, S. Jackson, R. R. Wilk, S. W. Englander, and W. F. DeGrado, Proteins 24 (1996) 427-32.

18 H. Wendt, L. Leder, H. Harma, I. Jelesarov, A. Baici, and H. R. Bosshard, Biochemistry 36 (1997) 204-13.

19 H. Wendt, A. Baici, and H. R. Bosshard, J. Amer. Chem. Soc. 116 (1994) 6973-74.

20 J. A. Zitzewitz, O. Bilsel, J. Luo, B. E. Jones, and C. R. Matthews, Biochemistry 34 (1995) 12812-9.

21 I. Jelesarov, E. Durr, R. M. Thomas, and H. R. Bosshard, Biochemistry 37 (1998) 7539-50.

22 E. Durr, I. Jelesarov, and H. R. Bosshard, Biochemistry 38 (1999) 870-80.

23 S. Ozeki, T. Kato, M. E. Holtzer, and A. Holtzer, Biopolymers 31 (1991) 957-66.

24 E. K. O'Shea, R. Rutkowski, and P. S. Kim, Science 243 (1989) 538-42.

25 J. P. L. J. D. D. W. F. Schneider, J. Amer. Chem. Soc. 119 (1997) 5742. 
26 G. B. Fields, Z. Tian, and G. Barany, in Synthetic Peptides A User's Guide, edited by G. A. Grant (W. H. Freeman and Company, 1992), pp. 126.

27 G. H. H. Olah, J. Chem. Phys. 89 (1988) 2531.

28 H. P. Lu and X. S. Xie, Nature 385 (1997) 143.

29 J. K. Trautman, J. J. Macklin, L. E. Brus, and E. Betzig, Nature 369 (1994) 40.

30 T. Ha, T. Enderle, D. S. Chemla, P. R. Selvin, and S. Weiss, Phys. Rev. Lett. 77 (1996) 3979.

31 T. Ha, T. Enderle, D. S. Chemla, P. R. Selvin, and S. Weiss, Chem. Phys. Lett. 271 (1997) 1.

32 T. Basche, S. Kummer, and C. Brauchle, Nature 373 (1995) 132.

33 M. A. Bopp, Y. Jia, G. Haran, E. A. Morlino, and R. M. Hochstrasser, Appl. Phys. Lett. 73 (1998) 7-9.

34 L. Streyer, Ann. Rev. Biochem. 47 (1978) 819-846.

35 D. S. Talaga, W. L. Lau, Y. Jia, H. S. M. Lu, W. DeGrado, and R. M. Hochstrasser, "Time Correlations Associated with Single Peptide Folding/Unfolding Dynamics" (To be published). 


\section{Figure Captions}

Figure 1. Schematic representation of the folding of GCN4-Pf. The right panel shows the crystal structure of folded GCN4-P1 with a hypothetical unfolded structure at the left. The peptide adheres to the positively charged surface by electrostatic interaction with the negatively charged glutamic acids at the $\mathrm{C}$ terminus of the peptide.

Conformational fluctuations cause changes in the donor-acceptor distance resulting in an anticorrelated modulation in the donor and acceptor fluorescence intensities.

Figure 2. CD spectra of GCN4-Pf in solution ( $2.0 \mathrm{mM})$, and adsorbed on modified quartz (surface density approximately $8 \mathrm{~nm}^{2} /$ molecule). The buffer is $10 \mathrm{mM}$ MES pH 6.1 .

Figure 3. Urea unfolding curve for surface-adsorbed GCN4-Pf. The mean residue ellipticity (MRE) at $222 \mathrm{~nm}$ was measured as a function of urea concentration, as described in Experimental. The surface density of the peptide on the quartz surface is approximately 1 molecule per $8 \mathrm{~nm}^{2}$.

Figure 4. Images of the donor (left) and acceptor (right) fluorescence of GCN4-Pf in the absence of denaturant at $\mathrm{pH}$ 6.1. Notice that the acceptor channel is much brighter than the donor channel.

Figure 5. Images of the donor (left) and acceptor (right) fluorescence taken at a urea concentration of $7.4 \mathrm{M}$ at $\mathrm{pH} 6.1$. The addition of denaturant has increased the donor channel intensity markedly with respect to the acceptor channel. 
Figure 6. Typical single molecule trajectories. The signals in the donor and acceptor channels are labeled R6G and TxR, respectively. The trajectories (a), (b) and (c) are discussed in the text.

Figure 7. High time resolution detection of donor (solid line) and acceptor (dashed line) fluorescence signals from a single GCN4-Pf molecule at $\mathrm{pH} 6.1$

Figure 8. Probability of occurrence $\mathrm{P}\left(\emptyset_{\mathrm{ET}}\right)$ of energy transfer efficiency $\emptyset_{\mathrm{ET}}$ versus $\varnothing_{\mathrm{ET}}$. The three rows correspond to urea concentrations 0,3 and $7.4 \mathrm{M}$. The three columns correspond to averaging times of 3,25 and $100 \mathrm{~ms}$. The solid line in the $3 \mathrm{M}$ urea distributions corresponds to an average of the 0 and $7.4 \mathrm{M}$ distributions for that time resolution.

Figure 9. Probability distributions $\mathrm{P}_{\mathrm{F}}(\mathrm{R})$ and $\mathrm{P}_{\mathrm{U}}(\mathrm{R})$ of the average donor acceptor separation, R, for the folded (F) and unfolded states (U).

Figure 10. Potentials of mean force for folded, $V_{F}(R)$, and unfolded, $V_{U}(R)$, states of GCN4-Pf on silanized glass. The potentials were calculated from $V(R)=-k_{B} T \ln$ $P(R)$, where $P(R)$ is the relevant experimentally determined probability distribution of donor acceptor distances. 


\section{Acknowledgements}

This work was supported by GM54616 (to WFD), GM12592 (to RMH) and GM48130 (to WFD and RMH) with instrumentation developed under RR01348.

D. Talaga was supported by NIH NRSA F32-GM18589. 


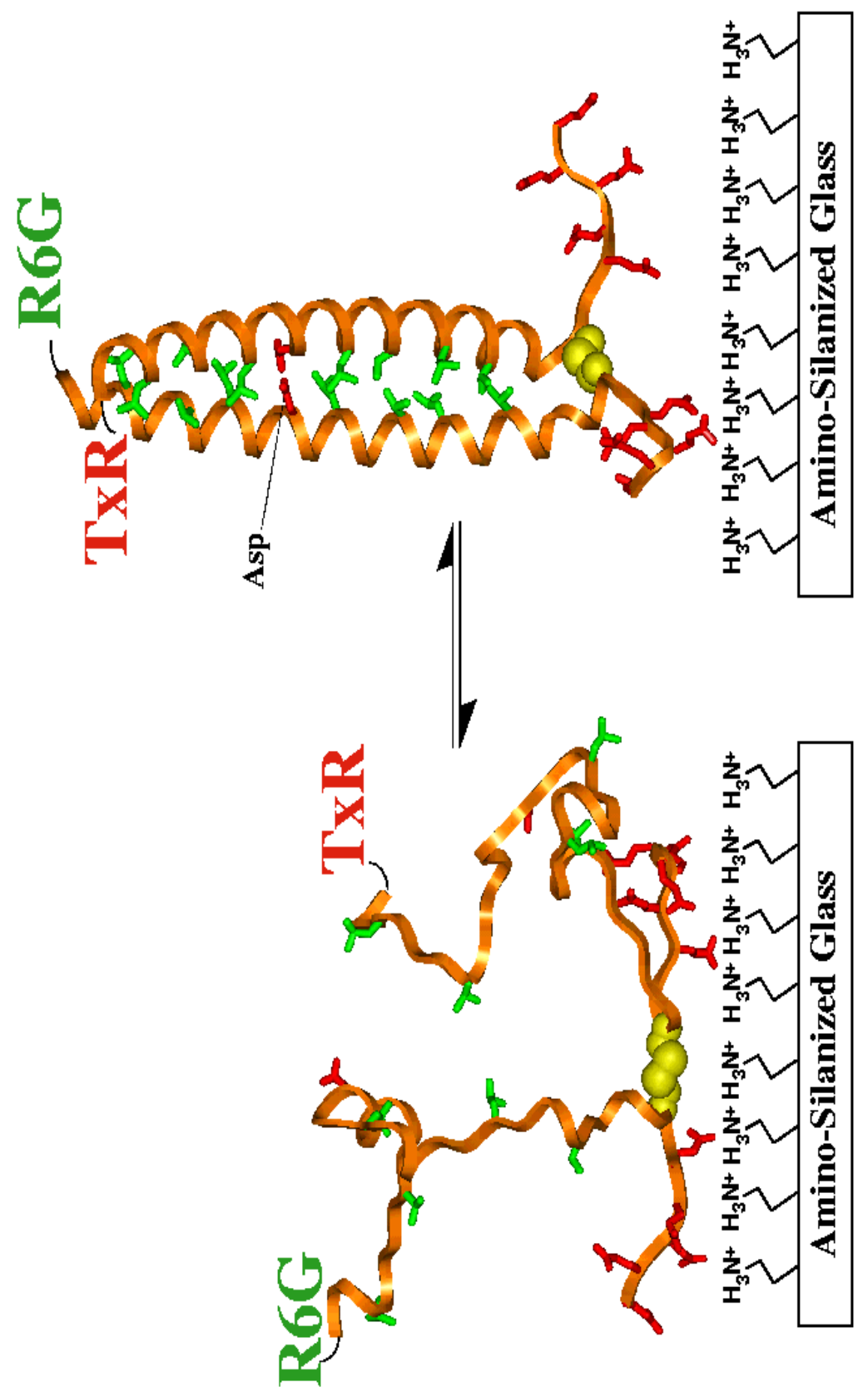

Fig. 1 


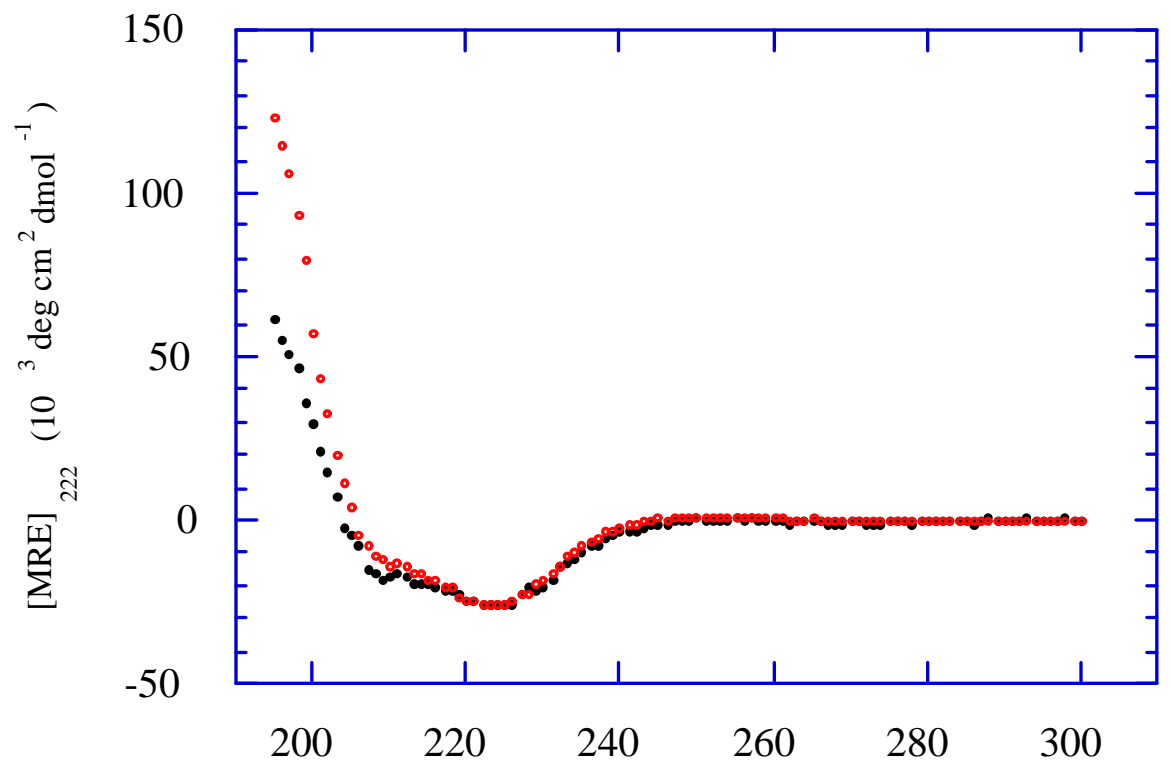




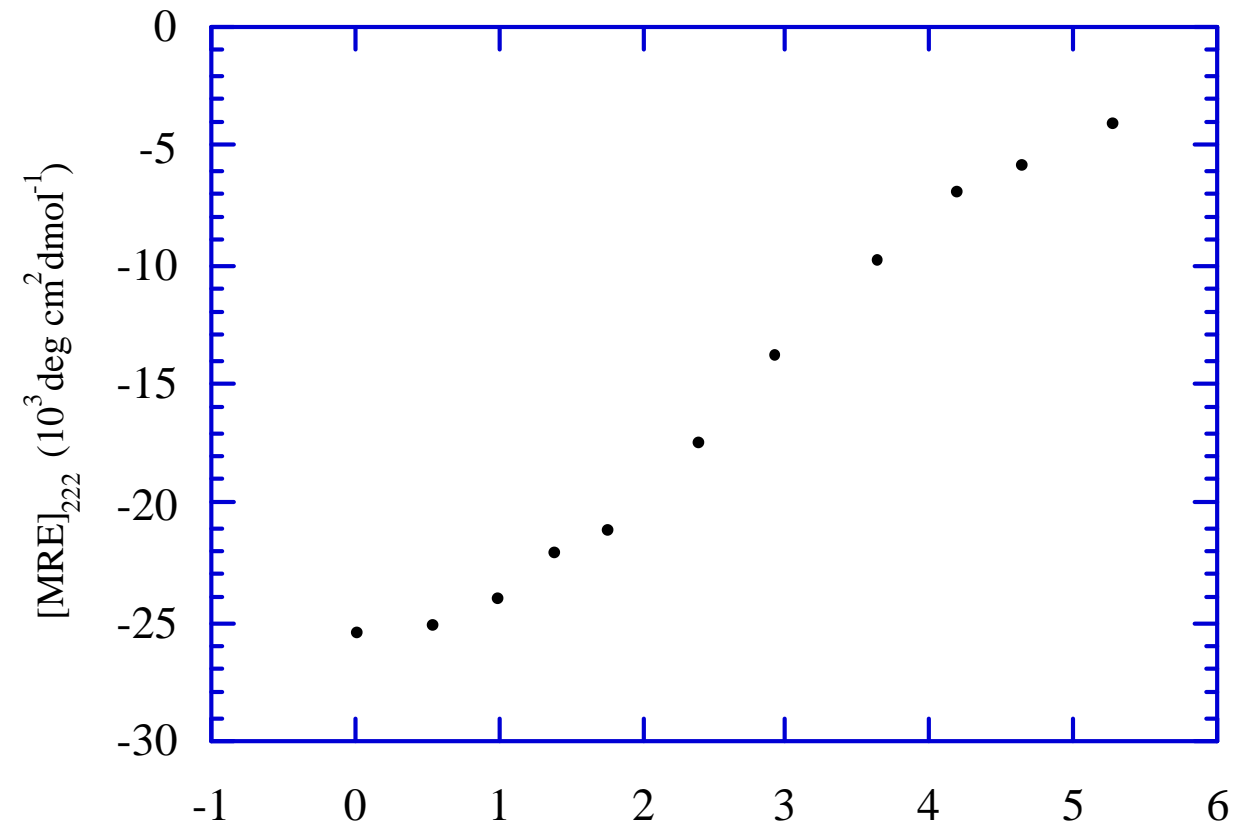




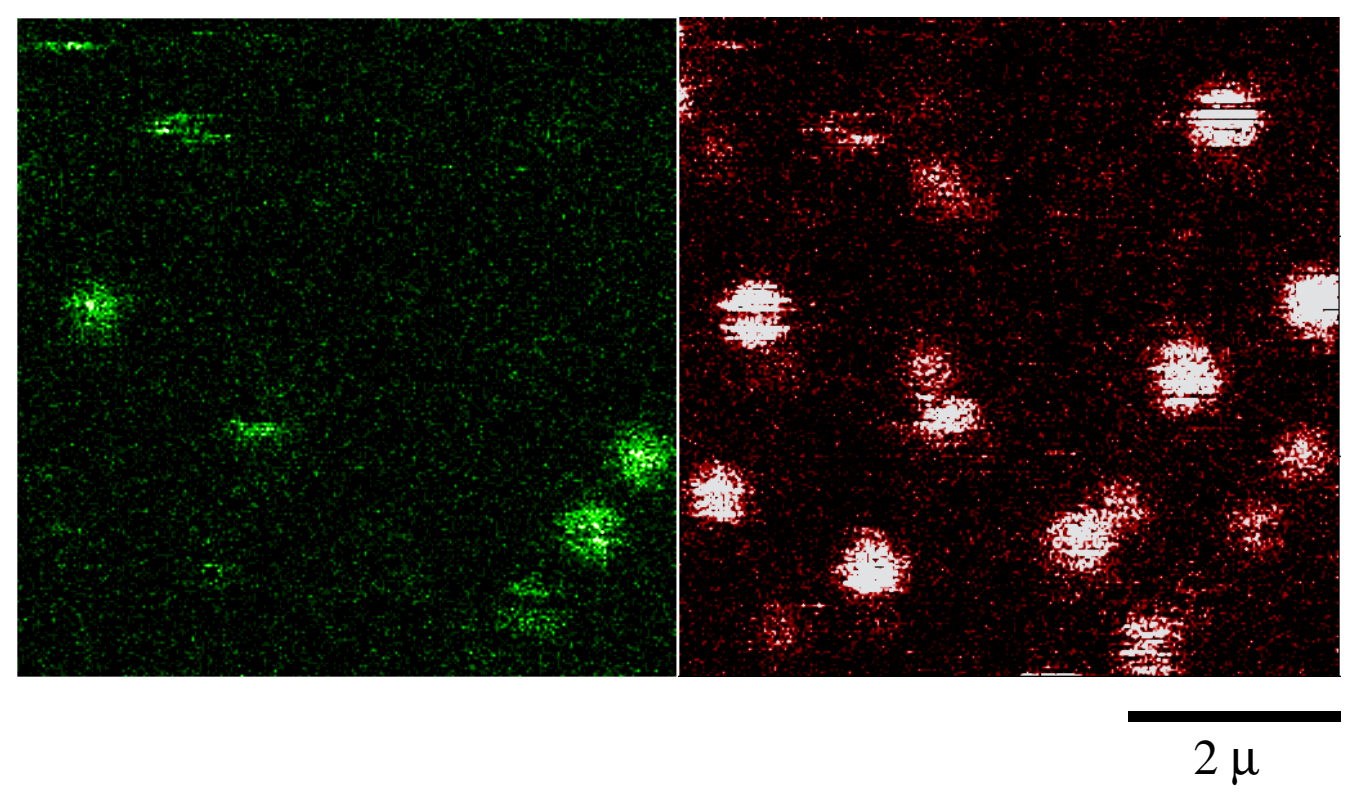




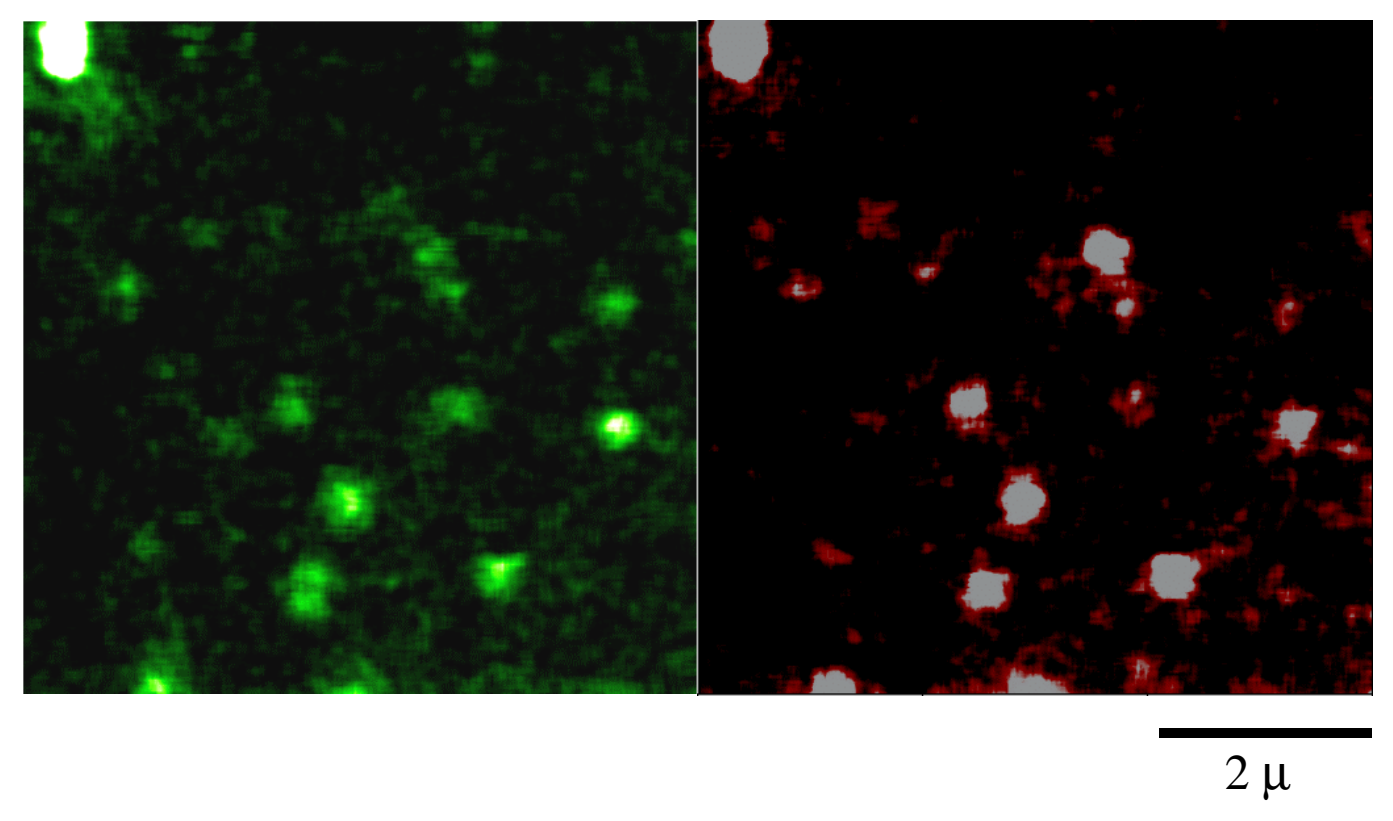



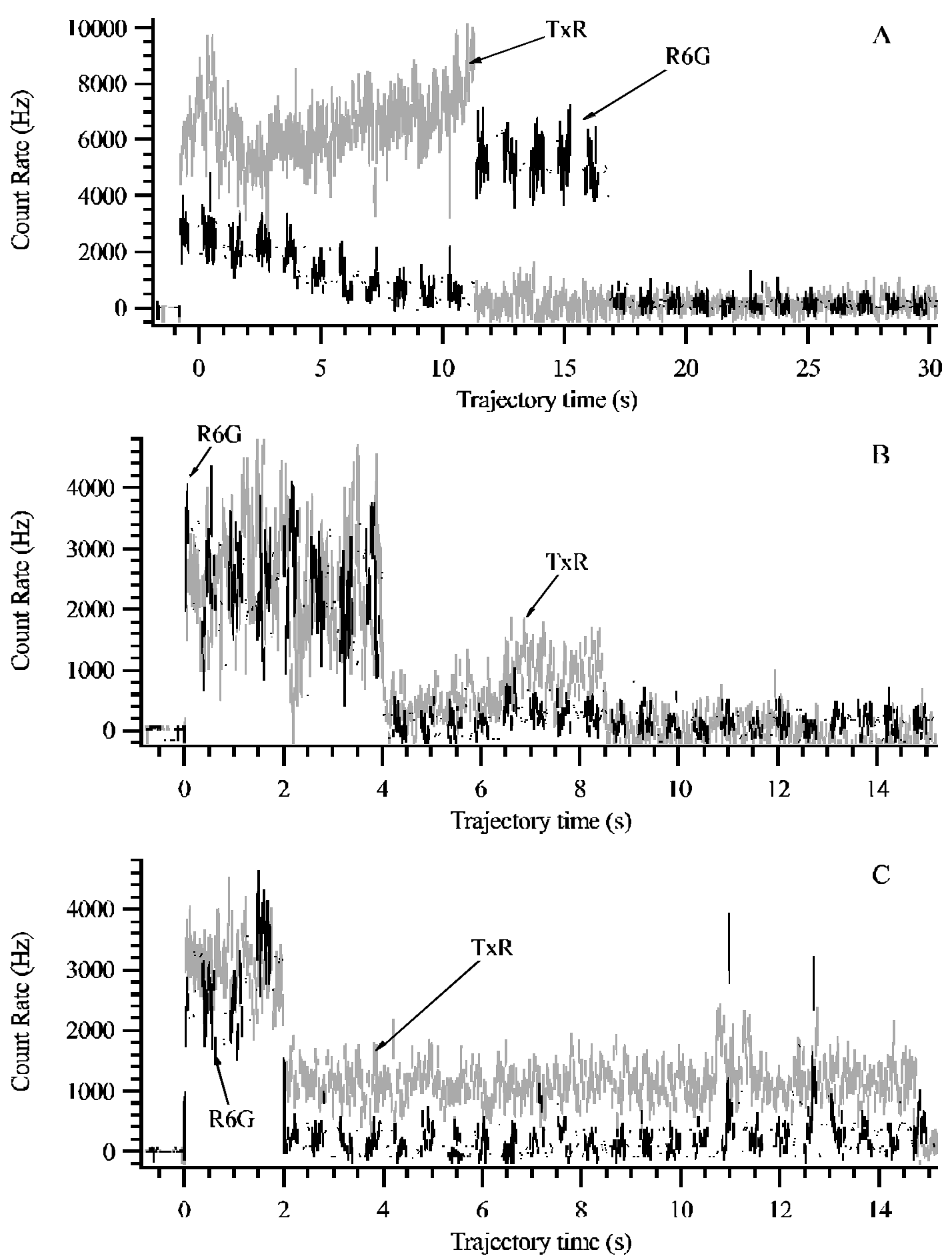


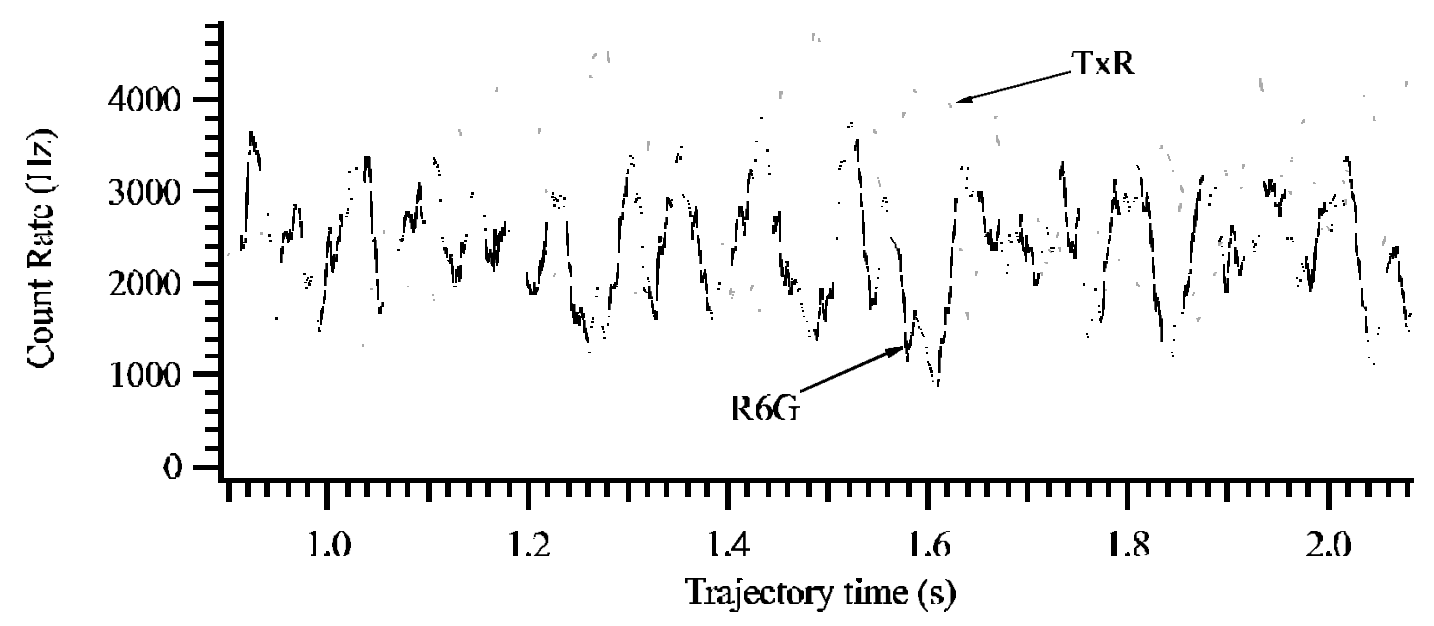




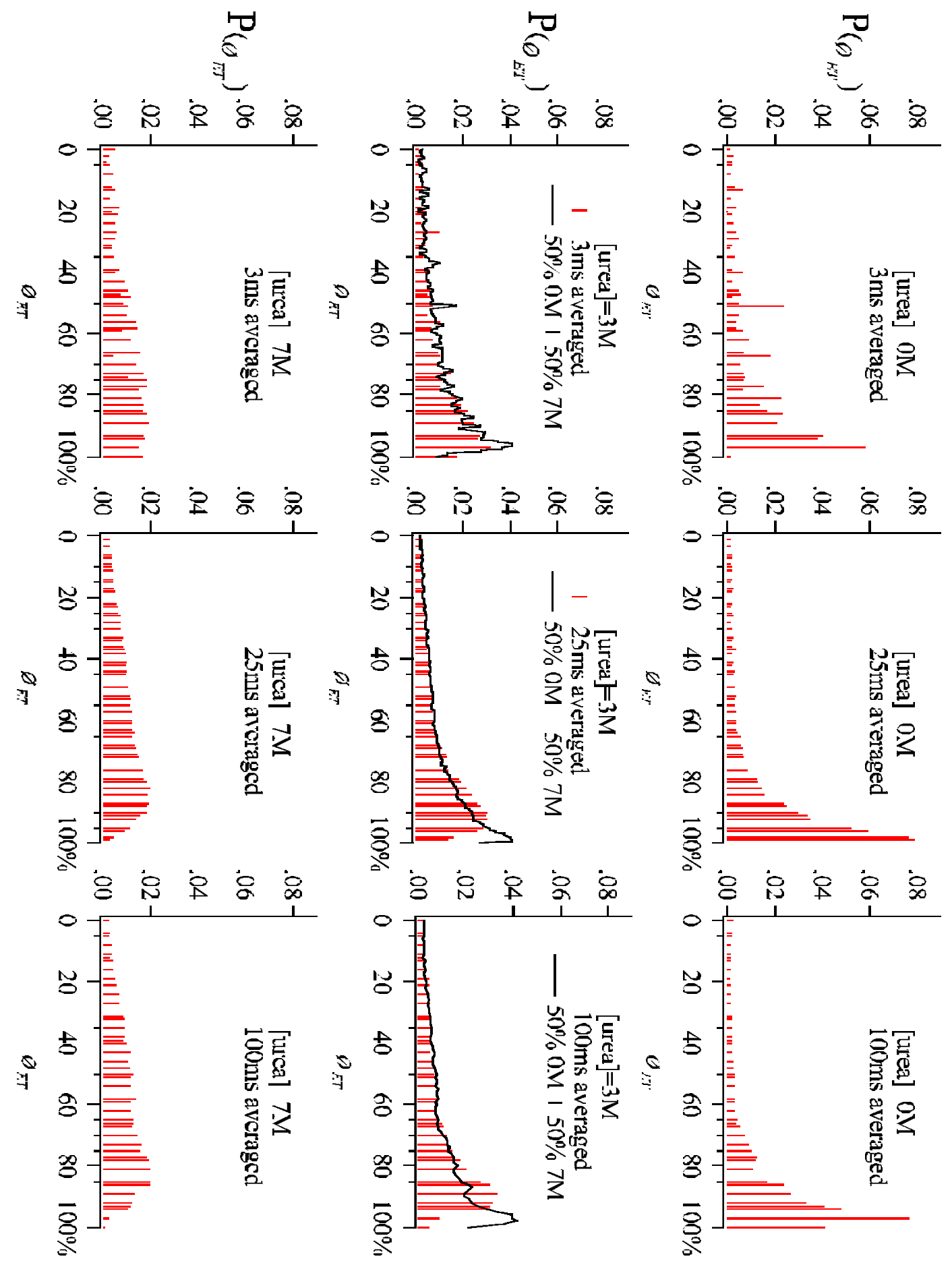



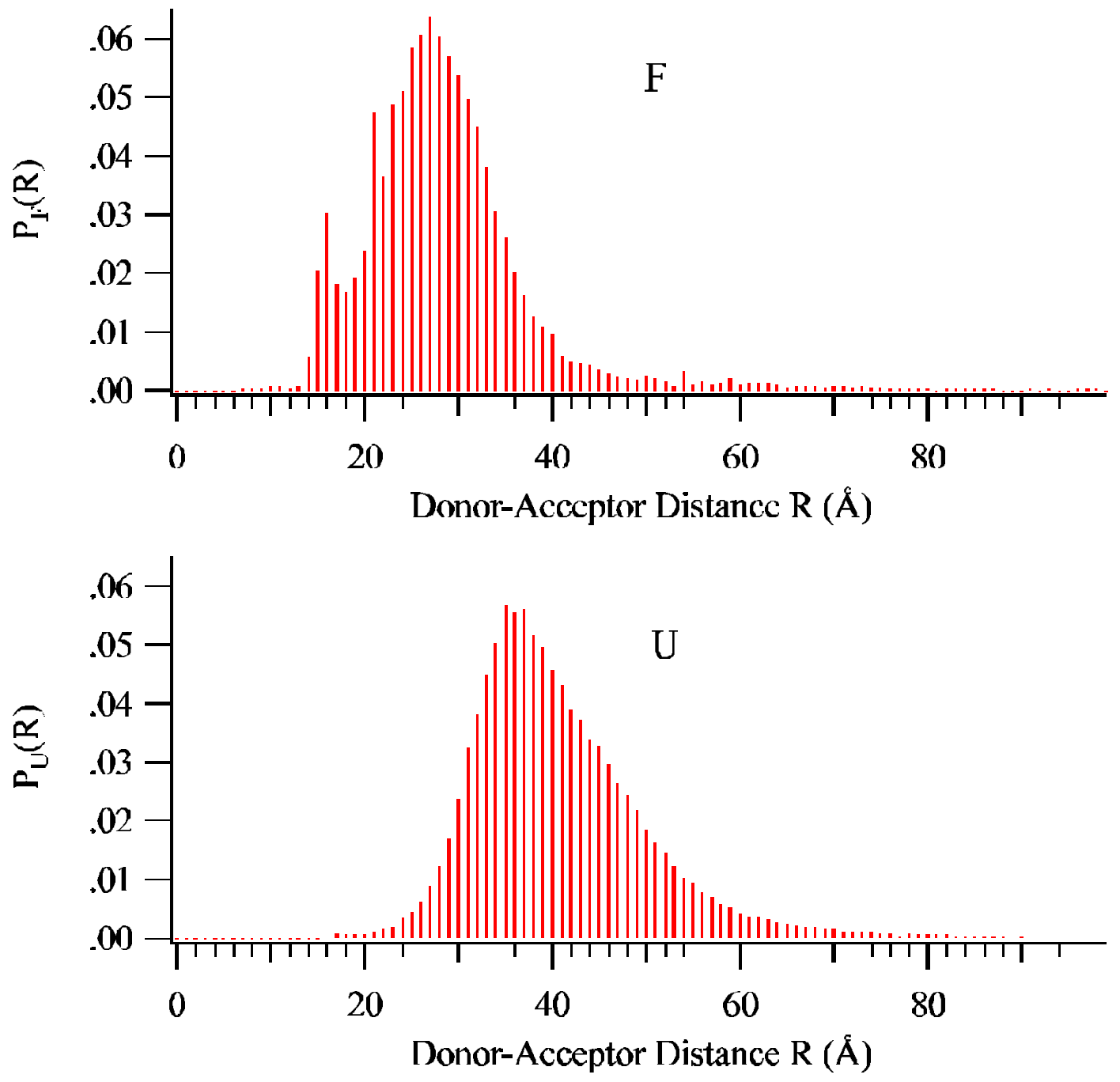


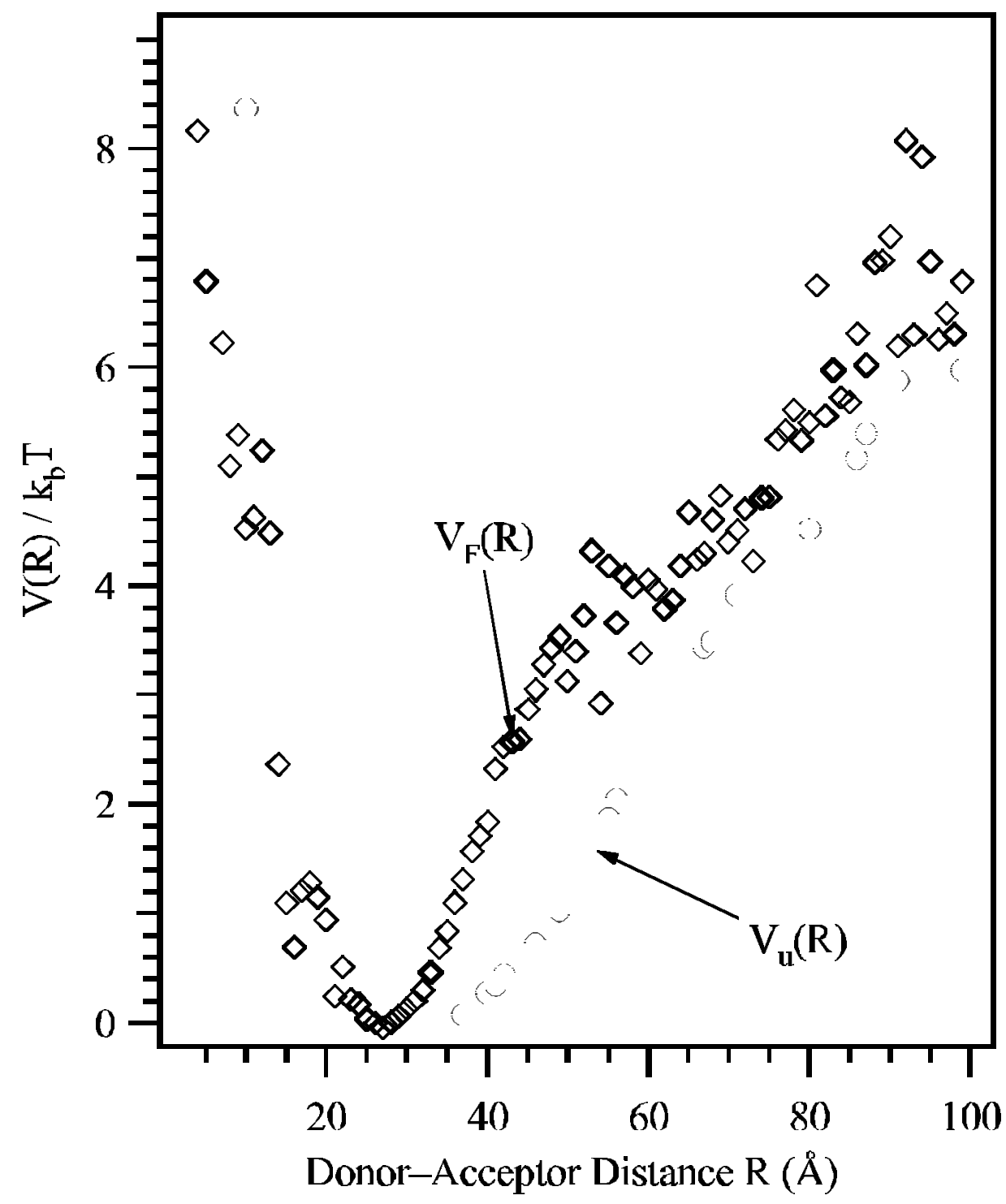


